\title{
The first year of open-access scientific journal publishing: our mishaps, our expectations, our plans for the bright future
}

\author{
by Yuri V. Artamonov#
}

1. Globustar Formaat, Harju maakond, Lasnamäe linnaosa, Valukoja tn 8/1, Tallinn, 11415, Estonia

\# Correspondence: yvartamonov@sbpreports.com

Received: 29.12.2021

Accepted: 30.12 .2021

Published: 30.12 .2021

DOI: $10.52455 /$ sbpr.01.202104013

\section{Why did we create SBPReports?}

In silico methods have become a versatile and effective tool for studying natural phenomena at multiple levels. However, unlike economics and physics, biology and physiology have always been slightly reluctant to accept the novel systemic approaches. Since scientific articles are among the critical metrics for the modern scientific society, the number of scientific journals devoted to some topic can be an excellent marker to evaluate the impact on society. Indeed, there are esteemed journals, such as PLOS Computational Biology or Journal of Theoretical Biology. Still, they focus mainly on a common biological phenomenon, while questions of physiology and pathophysiology are often overlooked.

On the other hand, the amount of work on computational biology is steadily growing, with $\sim 12000$ results published in 2010 and $\sim 28000$ in 2020 with the keyword "computational model", according to PubMed. With all this being said and our significant experience in the different aspects of computational physiology, our team has decided that it would be a mistake to lose an opportunity to cover this niche. Likewise, as a group of fellow scientists, at the end of 2019 , we decided to create our venture and launch our scientific journal - Systems Biology and Physiology Reports (SBPReports) [1]. This publisher's note would be an attempt to underline our first hectic year of open access scientific publishing.

\section{The first year of journal operation. Are we} successful?

The only effective way to check whether your venture is doing any good is to compare yourself to your successful competitors' performance in the first year of operation. For example, one of the most esteemed systems biology journals - PLOS Computational biology - has published more than 75 articles during its first year. It is an impressive number, in contrast to which our 15 articles appear to be relatively modest. However, PLOS Comp. Biol. is backed up by one of the pioneers in Open Access publishing - the Public Library of Science. Having such a powerful "investor" within your "venture" significantly facilitates your smooth start in the new field. On the other hand, the International Journal of Molecular Sciences - the flagship open-access journal of the MDPI Publishing - has also started rather modestly with 14 papers in four issues. It is also important to mention that in those years, MDPI was a very young publisher with barely any experience. Nowadays IJMS and MDPI are one of the most recognizable names in scientific publishing. Thus, it is safe to say that in terms of article numbers, our start has been rougher than we expected, yet some space for reasonable optimism is still present.

The birth of the journal and its first steps through the first year of existence

\section{Our strategy}

Before starting a new journal, SBPReports editors had a set of important choices to make. Above all was the selection of the journal publishing model and searching for the publisher. The answer to the first question was relatively simple. Only the open access publishing model is viable as no paywalls can be present in modern science. The second question, though, was much more complicated. On the one hand, major publishing houses, such as Elsevier or Wiley, provide significant support and give a tangible boost to a young journal. On the other hand, large companies do not like experiments. So, editors were afraid that they would not be able to imply all of the ideas into our journal with the support of such significant publishers. At that moment, SBPReports and Globustar Formaat have made contact, and our publishing team has guaranteed SBPReports editors reasonable freedom in terms of the development strategy of a journal. After one year of SBPReports existence, we cannot say that this was the most straightforward choice, yet we believe that certain independence is the key to the sustainable growth of the journal.

\section{From the very beginning}

So, as an editorial board was formed and partnership agreement was made with the publisher, SBPReports was ready to go. Initially, we had five 
sections in the journal and aimed to publish at least a paper in each section quarterly. However, no one said that launching a journal from scratch would be easy, and we started to face difficulties from the first moments as we planned to publish at least five papers in every issue. However, it appears that there are not that many enthusiasts that would send their work to the unknown journal without an impact factor and not present in the Scopus or Web of Science. Furthermore, the list of the requirements of these databases is strict, and above all, we would have to be operational for more than two years to apply. On the other hand, we had to be very accurate in selecting the works we would publish as we aim to the upper level of journals. After one year, not much can be said on how effectively we cope with all of the difficulties as only citations and overall recognition in the society would give us the desired answers. Unfortunately, these are long-term markers, but we need to stay optimistic and remain firmly on our principles. Our ambition remains to create a forum for in-depth discussions between physiologists, physicians, biologists, biochemists, biophysicists, and mathematicians that would be understandable and meaningful for all those fields. This does not allow us to make any compromises in terms of selecting what to publish. Initially, we aimed to be eligible for inclusion into all databases within the two years of publishing. We still desire this to happen, but we have to be realistic and agree that this is an excessively optimistic scenario with the current tempo of paper publishing. However, we believe that inclusion into the Russian Science Citation Index database would give us an additional boost and increase the inflow of manuscripts. Alongside $\mathrm{RSCl}$, we aim into the Directory of Open Access Journals, which requires 20 articles from the new journal. We believe that these steps would be of essential importance for us on our path to Scopus and the Web of Science.

\section{SBSP conferences and a platform for supporting the conferences}

It would be a significant omission not to mention creating a conference platform based on SBPReports. The starting point was the beginning of cooperation with the organizers of the virtual conference Systems Biology and Systems Physiology (SBSP) in December 2020. At that time, we only published the abstracts of the forum. In the summer of 2021, the organizers decided to hold the SBSP 2021 conference dedicated to the Anniversary of Fazly Ataullakhanov in a hybrid format, which required the creation of a multifunctional platform. Based on SBPReports, a platform was created that allowed to track the schedule of reports, take a look at conference materials (texts of abstracts, video recordings of oral communications) etc.

\section{Conclusion}

All of these excellent research and review studies, alongside the topics addressed during Systems Biology and Systems Physiology conferences, are the core that shapes the scope of the SBPReports for the following years. Undoubtedly, the first year was tough, and much has not been following our initial strategic plans. However, we believe that our dedication and commitment should be the basis for the future flourishing of the SBPReports. In the following year, we aim to at least double the number of published papers and hold one more SBSP congress on the intracellular signaling in platelets. Among other vital targets is increasing our social media presence and entering the first scientific databases - RSCl and DOAJ.

On behalf of the SBPReports publishing team Globustar Formaat CEO

Yuri V. Artamonov

\section{References}

1. Panteleev M. Systems approaches meet biology and physiology: why do we need yet another journal? Systems Biology and Physiology Reports 2021;1:1. https://doi.org/10.52455/sbpr.01.202101011. 